\title{
Sharp Infrared Emission from Single-Crystalline Indium Nitride Nanobelts Prepared Using Guided-Stream Thermal Chemical Vapor Deposition**
}

\author{
By Ming-Shien Hu, Wei-Ming Wang, Tzung T. Chen, Lu-Sheng Hong, Chun-Wei Chen, Chia-Chun Chen, \\ Yang-Fang Chen, Kuei-Hsien Chen, and Li-Chyong Chen*
}

Single-crystalline InN nanobelts have been synthesized using Au as the catalyst by a guided-stream thermal chemical vapor deposition technique. The resultant InN nanobelts typically have widths ranging from 20 to $200 \mathrm{~nm}$, a width to thickness ratio of $2-10$, and lengths of up to several tens of micrometers. Structural analysis shows that these InN nanobelts have a wurtzite structure and exhibit a rectangular cross section with self-selective facets, i.e., the nanobelts are enclosed only by $\pm(001)$ and $\pm(1 \overline{1} 0)$ planes with [110] being the exclusive growth direction along their long axis. This facet selectivity can be understood by the differences in the surface energies of the different facets. Photoluminescence (PL) spectra of InN nanobelts show a sharp infrared emission peak at $0.76 \mathrm{eV}$ with a full width at half maximum of $14 \mathrm{meV}$, narrower than the values reported for InN epilayers. The integrated PL intensity is found to increase linearly with the excitation power, which suggests that the observed PL can be attributed to direct band-to-band emission.

\section{Introduction}

Amongst group-III nitride semiconductors, InN has been predicted to have the lowest effective mass, ${ }^{[1]}$ which would lead to a high mobility and high saturation velocity, making it suitable for applications in high-speed and high-frequency electronic devices. Recently, InN has attracted much attention ow-

[*] Dr. L. C. Chen

Center for Condensed Matter Sciences

National Taiwan University

Taipei 106 (Taiwan)

E-mail: chenlc@ccms.ntu.edu.tw

M. S. Hu, Prof. L. S. Hong

Department of Chemical Engineering

National Taiwan University of Science and Technology

Taipei 106 (Taiwan)

M. S. Hu, Dr. K. H. Chen

Institute of Atomic and Molecular Sciences

Academia Sinica

PO Box 23-166, Taipei 106 (Taiwan)

W. M. Wang, Prof. C. C. Chen

Department of Chemistry

National Taiwan Normal University

Taipei 106 (Taiwan)

T. T. Chen, Prof. Y. F. Chen

Department of Physics

National Taiwan University

Taipei 106 (Taiwan)

Prof. C. W. Chen

Department of Material Science and Engineering

National Taiwan University

Taipei 106 (Taiwan)

[***] This research was financially supported by the Ministry of Education in Taiwan. The authors thank Dr. S. Chattopadyay for fruitful discussions. ing to the heated debate over the reversion of the fundamental bandgap from $1.8-2.1 \mathrm{eV}$ to $0.7-0.8 \mathrm{eV} .^{[2]}$ With the newly suggested bandgap of InN $(0.7-0.9 \mathrm{eV})$, group-III-nitride alloybased light-emitting diodes could possibly be operated at spectral wavelengths ranging from the near-infrared to the ultraviolet region of the electromagnetic spectrum $(0.7-6.2 \mathrm{eV})$. With GaN having been the focus of attention for over a decade, the development and understanding of InN has lagged behind. Currently, the synthesis of high-quality $\mathrm{InN}$ is still a challenge because of the extremely high equilibrium vapor pressure of nitrogen and the low decomposition temperature of $\operatorname{InN}^{\left[{ }^{[3]}\right.}$

Most studies of InN, including studies of crystal growth, physical properties, and device fabrication, have been primarily focused on $\mathrm{InN}$ in thin-film forms. InN-based nanostructures, such as nanocrystals, ${ }^{[4]}$ quantum dots, ${ }^{[5]}$ nanowires, ${ }^{[6]}$ nanorods, ${ }^{[7]}$ and nanotubes, ${ }^{[6 \mathrm{~d}, 8]}$ have started receiving growing attention because of the great potential they offer for studying the dependence of various physical properties on dimensionality and size. One-dimensional (1D) nanostructures are unique because they exhibit a discrete density of states and at the same time maintain a continuous transport path. Although a few groups have been successful at fabricating $1 \mathrm{D}$ InN nanostructures, ${ }^{[6-9]}$ to the best of our knowledge, InN nanobelts have never been reported before.

Structures with a rectangular cross section are often called nanobelts or nanoribbons, ${ }^{[10]}$ and their well-defined faceted nature enables the observation of unique optical-confinement and microcavity effects. ${ }^{[1]}$ According to classical waveguide theory, waveguides of different cross sections will exhibit different transverse optical (TO) modes. ${ }^{[12]}$ Nanobelts with rectangular cross sections have been used as effective Fabry-Perot microcavities for lasing. ${ }^{[13]}$ In this article, we report the successful syn- 
thesis of wurtize InN nanobelts using a guided-stream thermal chemical vapor deposition (GSCVD) technique. Moreover, the GSCVD-prepared InN nanobelts exhibit a sharp emission peak at $0.76 \mathrm{eV}$ with a full width at half maximum (FWHM) of $14 \mathrm{meV}$, the smallest value reported for InN so far. Such a sharp peak is remarkable as typical FWHM values reported for highquality epitaxial InN films range from 20 to $70 \mathrm{meV} .^{[14]}$

\section{Results and Discussion}

After growth, black materials are found to cover the entire surface of the substrate $(0.5 \mathrm{~cm} \times 0.5 \mathrm{~cm})$. Figure 1 shows typical field-emission scanning electron microscopy (FESEM) images of the as-synthesized product. The low-magnification image shown in Figure 1a reveals that the product consists of belt-like structures with typical lengths of a few tens of micrometers. High-magnification images (Figs. 1b,d) show the morphology of the nanobelts, with widths ranging from 20 to $200 \mathrm{~nm}$ (Fig. 1c), and the thickness ranging from half to one tenth of the width.

The X-ray diffraction (XRD) pattern of the product is shown in Figure 2a. All the reflections can be indexed to $\mathrm{InN}$ with a hexagonal structure. The lattice constants calculated from the diffraction pattern are $a=0.355 \mathrm{~nm}$ and $c=0.573 \mathrm{~nm}$, in good agreement with the standard literature values of $a=0.354 \mathrm{~nm}$ and $c=0.571 \mathrm{~nm}$ (Joint Committee on Powder Diffraction Standards (JCPDS) 02-1450). Figure 2b shows the Raman spectrum of these structures measured at room temperature. Apart for the Raman peak of $\mathrm{Si}$ at $520 \mathrm{~cm}^{-1}$, the peaks observed at 449,485 , and $554 \mathrm{~cm}^{-1}$ can be assigned to $\mathrm{A}_{1}$ (TO), $\mathrm{E}_{2}$, and $\mathrm{A}_{1}$ longitudinal optical (LO) phonon modes of $\mathrm{InN}$, respectively. ${ }^{[6,7]}$ In comparison with the reported values for highquality InN thin films, it is worth noting that the $\mathrm{A}_{1}$ (LO) peak
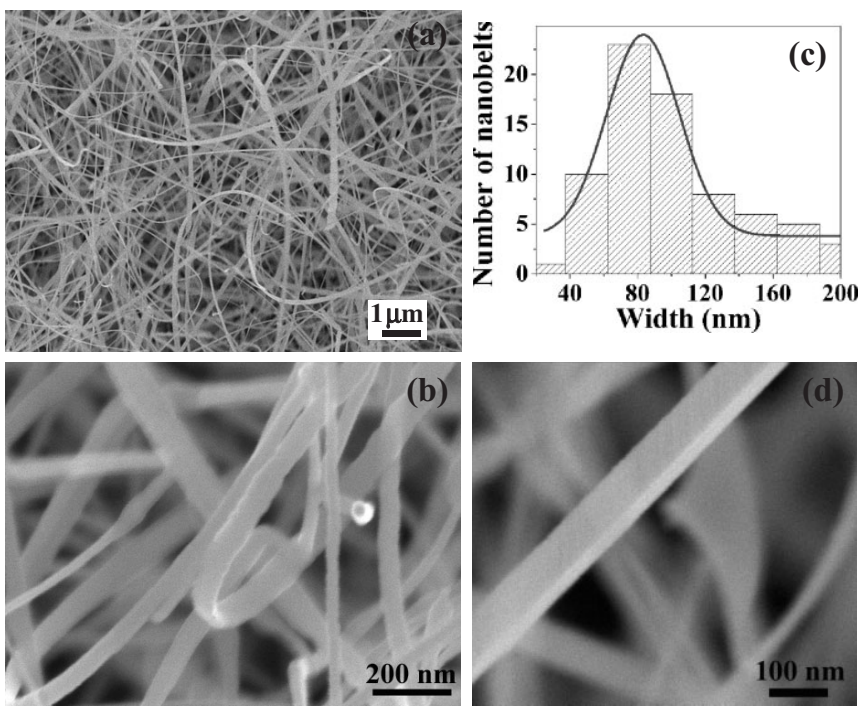

Figure 1. Typical FESEM images of InN nanobelts: a) Low-magnification SEM image. b) High-magnification SEM image of the as-synthesized products. c) Size distribution of InN nanobelts. d) Image showing the belt shape of a single nanobelt.
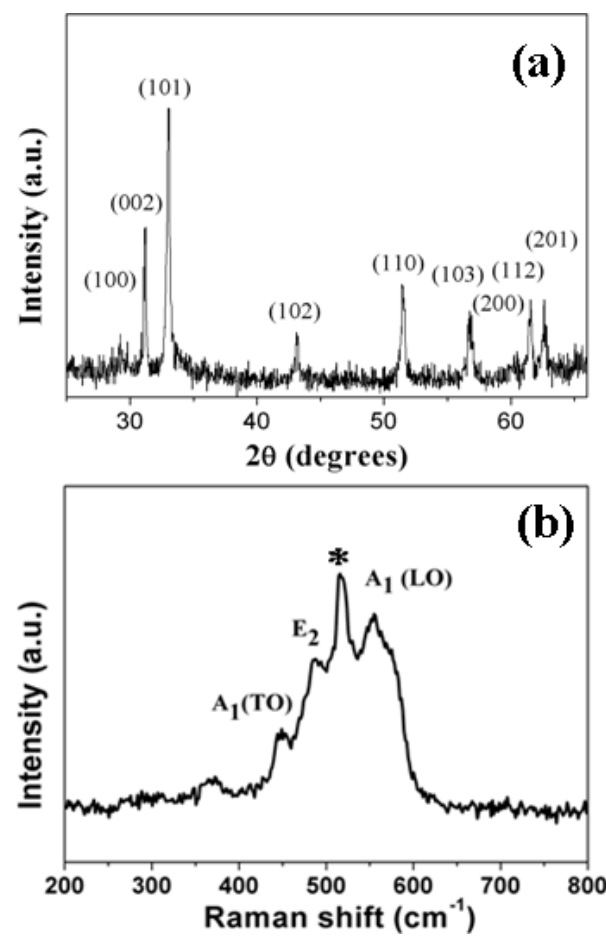

Figure 2. a) XRD pattern of as-prepared InN nanobelts. b) Typical Raman spectrum of InN nanobelts (* represents the signal from the Si substrate).

shows a clear shift towards lower wavenumbers. This result is similar to other nanowires and nanorods, and may be attributed to size confinement effects. ${ }^{[6,7]}$

The morphology and microstructure of the GSCVD-prepared InN nanobelts have been further characterized using transmission electron microscopy (TEM). Figures 3a,b show typical bright-field TEM images of the InN nanobelts. The dark stripes on the nanobelts result from bending contours, ${ }^{[15]}$ which are normally observed in TEM studies of bent thin crystals. The observation of a catalytic nanoparticle $(25 \mathrm{~nm})$ attached to the growth front (Fig. 3c) strongly suggests that the nanobelts are formed by a vapor-liquid-solid (VLS) mechanism. The corresponding energy-dispersive X-ray spectroscopy (EDX) spectrum (Fig. 3c, inset) confirms that most of the catalytic nanoparticles are composed of a $\mathrm{Au}-\mathrm{In}$ alloy with negligible $\mathrm{N}$ content. Figure $3 \mathrm{~d}$ is a high-resolution TEM (HRTEM) image taken at the edge of the nanobelt shown in Figure 3c. The inset to Figure 3d shows a typical selected-area electron diffraction (SAED) pattern of hexagonal InN recorded with a [001] zone axis. The observed interplanar spacing of the lattice planes is $0.308 \mathrm{~nm}$, which corresponds to the (100) lattice planes of hexagonal InN. The TEM study reveals that all the nanobelts grow along the [110] direction, and are enclosed exclusively by $\pm(001)$ and $\pm(1 \overline{1} 0)$ facets. This growth direction is identical to the predominant growth direction perpendicular to the (110) plane that has previously been reported for InN nanowires and nanorods. ${ }^{[6,7]}$ Furthermore, elemental analysis of a nanobelt (shown in Figs. 4b,c) using electron energy-loss spectroscopy (EELS) clearly indicates a stoichiometric composition with a negligible oxygen signal (Fig. 4d). 

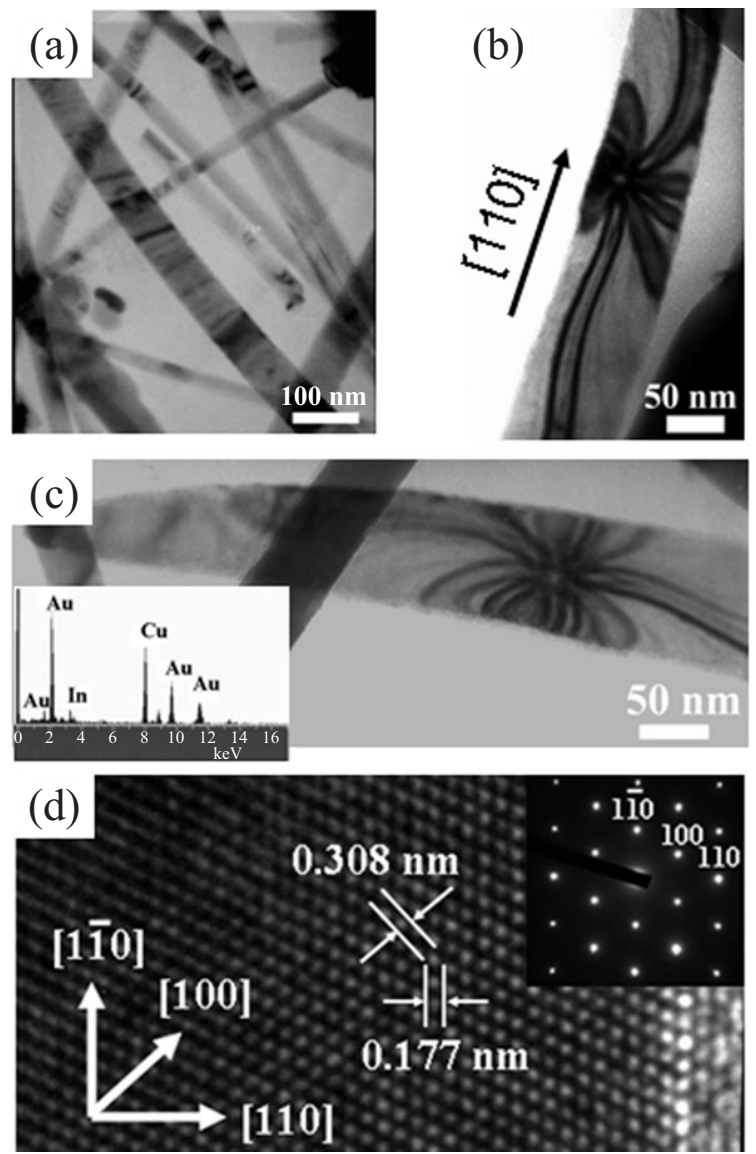

Figure 3. a,b) Low-magnification TEM images of $\ln N$ nanobelts. c) TEM image showing the presence of a nanoparticle (Au-In alloy) attached to the growth front of a nanobelt; inset: the EDX spectrum of the nanoparticle. d) HRTEM image and corresponding SAED pattern taken from a nanobelt with a [110] growth direction.
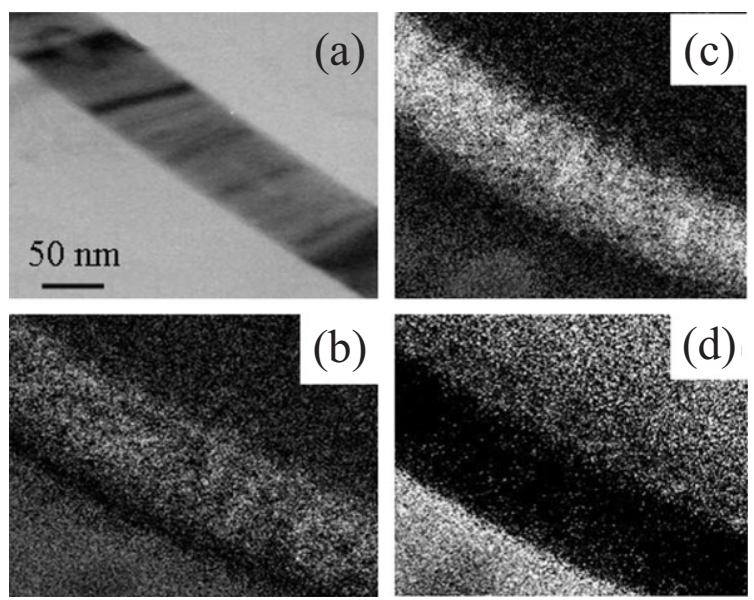

Figure 4. a) TEM image of a single InN nanobelt and corresponding EELS mapping of b) In, c) $\mathrm{N}$, and d) $\mathrm{O}$.

For the growth of nanobelts in various material systems, two types of growth mechanisms have been proposed, namely, the vapor-solid (VS) ${ }^{[10,15 b, 16]}$ and VLS ${ }^{[17]}$ mechanisms. In the VLS process, a metallic liquid droplet at the growth front can serve as a catalytic site, and thus kinetic processes most likely dominate the resultant morphology. ${ }^{[18]} \mathrm{InN}$ nanobelts grown by the newly developed GSCVD process show signatures of a VLS growth mechanism. The guided-stream technique has been used to accelerate the flow velocity of $\mathrm{NH}_{3}$, which is expected to reduce variations in the temperature and concentration of $\mathrm{NH}_{3}$ before the gas reaches the substrate (more details in the Experimental section). The growth temperature of the InN nanobelts is $560^{\circ} \mathrm{C}$, which is higher than that of $\mathrm{InN}$ nanowires or nanorods $\left(500^{\circ} \mathrm{C}\right)$. This indicates that the growth of $\mathrm{InN}$ nanobelts requires more efficient dissociation of $\mathrm{NH}_{3}$ in the gas phase to generate more nitrogen-containing radicals.

It has been reported that the morphologies of nanobelts are governed by a combination of surface energy and growth kinetics. ${ }^{[18]}$ The surfaces of the nanobelts are dominated by low index planes, which may be attributed to their lower-energy surfaces. Figures 5a,b show unit cell models of InN along [001], [110], and [110] directions built using the CERIUS2 software package. ${ }^{[19]}$ These cleaved planes correspond to structural models of the nanobelt structure shown in Figure 5d. The number of broken bonds for each plane in each unit cell is seen to be one, two, and four, for (001), (11̄0), and (110) planes, respectively. Therefore, the number of broken bonds per unit area for each crystal plane is calculated to be $0.92 \mathrm{~nm}^{-1}$ for (001), $0.99 \mathrm{~nm}^{-1}$ for $(1 \overline{1} 0)$, and $1.14 \mathrm{~nm}^{-1}$ for (110). Thus, it can be qualitatively inferred that the surface energies $(\gamma)$ for these three surfaces follow the following trend: $\gamma_{(001)}<\gamma_{(1 \overline{1} 0)}<\gamma_{(110)}$, which is also consistent with other III-V nitrides. ${ }^{[20]}$ The (001) and (110) surfaces observed in the InN nanobelts may be attributed to their smaller surface energy, which also suggests that the surfaces of the nanobelts are enclosed by low-index,

(a)

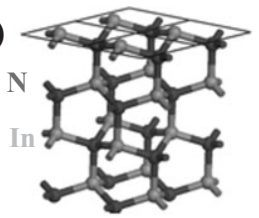

(001)

(b)

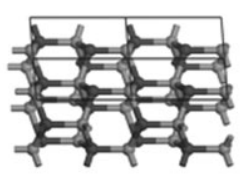

(110)

(c)

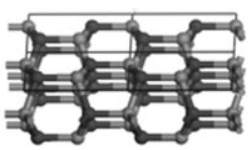

(110)

(d)

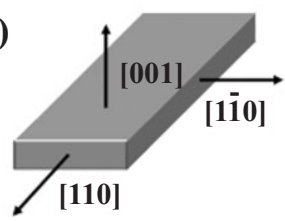

Figure 5. Unit-cell model of hexagonal $\ln \mathrm{N}$ constructed using CERIUS2 software: a) (001), b) (110), and c) (110). d) Schematic diagram of the nanobelt crystallographic directions. 
low-energy crystallographic facets. Furthermore, it is worth noting that all VLS-grown InN nanowires or nanobelts grow along the [110] direction, whereas VS-grown InN nanowires grow along the [001] direction. ${ }^{[6]}$ Ding et al. have recently reported that an individual metal nanoparticle can lead to two kinds of 1D nanostructures by the VLS mechanism, either nanobelts or nanowires. They conclude that the shape of the 1D nanostructure is determined by the interfacial lattice mismatch between the catalytic nanoparticle and the $1 \mathrm{D}$ nanostructure. ${ }^{[17 \mathrm{~b}]}$ It is believed that both the growth temperature and the modified flow stream play crucial roles in determining the shape of the nanobelts, although a detailed mechanism is yet to be understood.

Photoluminescence (PL) spectra of the nanobelts measured at $20 \mathrm{~K}$ (Fig. 6a) shows typical excitation power dependence. A strong PL emission at $0.76 \mathrm{eV}$ is observed. The integrated PL intensity increases linearly with the excitation power over one order of magnitude, and the peak position does not shift in this excitation energy range (Fig. 6b). This suggests that the PL arises from fundamental interband transitions of InN. ${ }^{[2,21]}$ The absence of PL signal saturation even at the highest excitation power of $200 \mathrm{~mW}$ further confirms that the emission arises from direct
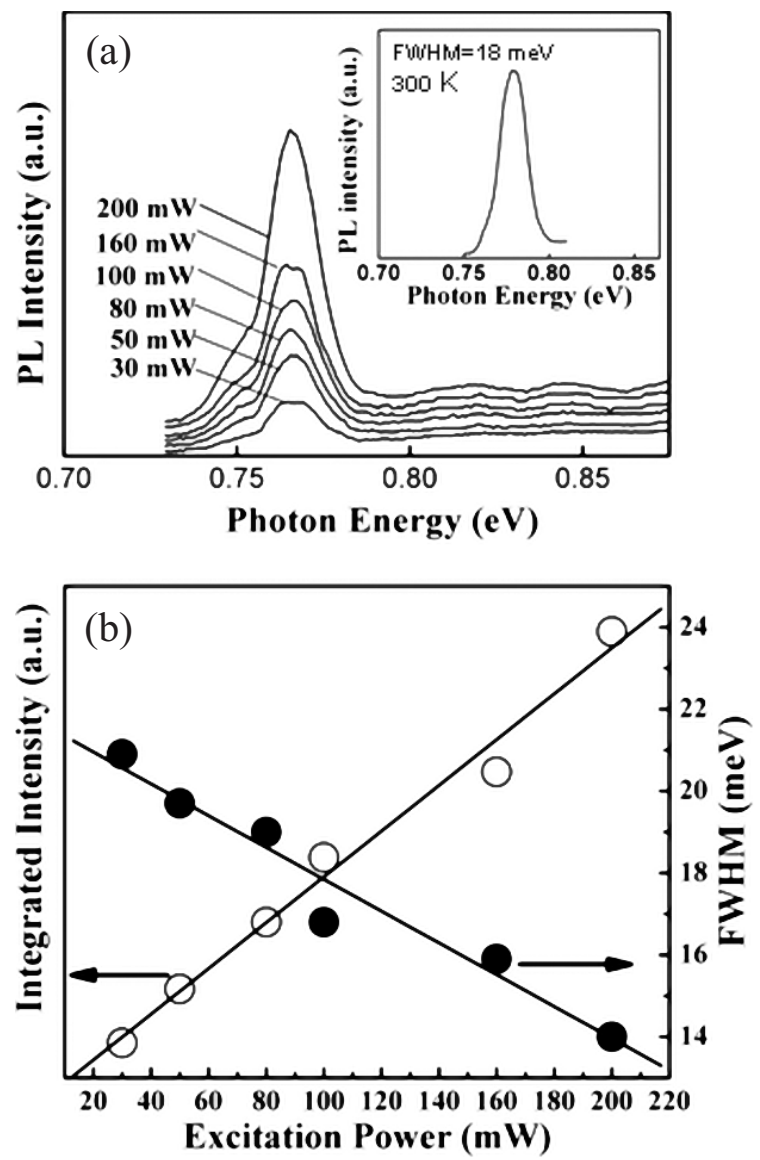

Figure 6. a) Power-dependent PL spectra of InN nanobelts recorded using a InGaAs detector at $20 \mathrm{~K}$; inset: the room-temperature PL spectrum at an excitation power of $100 \mathrm{~mW}$. b) Plots of integrated PL intensity and FWHM of the emission peak as a function of the excitation power, measured at $20 \mathrm{~K}$. band-to-band recombination. Furthermore, it is worth noting that the FWHM of the PL spectra is only $14 \mathrm{meV}$. As compared to the $\sim 20-70 \mathrm{meV}$ FWHM values typically observed for highquality molecular beam epitaxy (MBE)-grown InN epilayers, ${ }^{[2,14]}$ the linewidths of the PL spectra for InN nanobelts are remarkably narrow. The room-temperature $(300 \mathrm{~K}) \mathrm{PL}$ spectrum (inset to Fig. 6a) of InN nanobelts, recorded at an excitation power of $100 \mathrm{~mW}$, also shows a narrow FWHM of $18 \mathrm{meV}$. The linewidth of the emission peak is plotted as a function of the excitation power in Figure 6b. Progressive narrowing of the peak width (from $\sim 21$ to $14 \mathrm{meV}$ ), along with enhancement of the PL intensity, is observed as the excitation power is increased from 30 to $200 \mathrm{~mW}$. Strong optical-microcavity effects have been demonstrated previously in $\mathrm{ZnO}$ nanobelts. ${ }^{[13]}$ It is conjectured that amplified spontaneous emission may occur along the direction of the nanobelts as the pump power increases. This will lead to narrowing of the emission peak owing to the preferential amplification of photons close to the maximum of the gain spectrum. ${ }^{[22]}$ Similar narrowing of linewidths has also been observed in the optical pumping of a highly-scattering gain media consisting of nanoparticles dispersed in either a dye solution or a solid host. ${ }^{[23]}$ In addition, a visible-wavelength PL study was carried out using a separate micro-PL setup (Renishaw 2000), and no PL signal was seen around $1.9 \mathrm{eV}$.

The observation of strong PL of the InN nanobelts in only the infrared region, and not in the visible region of the electromagnetic spectrum, is consistent with results for high-quality single-crystalline InN films grown by MBE. In contrast, for InN films containing very fine grains, a substantial blue-shift of the PL peak has been reported, ${ }^{[24]}$ which can be accounted for by quantum-size effects, owing to the small effective mass of InN. ${ }^{[25]} \mathrm{PL}$ emission at $1.85 \mathrm{eV}$ from oxygen-free InN nanowires with average diameters smaller than $30 \mathrm{~nm}$ have also been reported, while a PL peak at $\sim 0.77 \mathrm{eV}$ is seen for nanowires with diameters exceeding $50 \mathrm{~nm} \cdot{ }^{[6,7]}$ From effective-mass approximation analysis, a pronounced quantum-size effect is expected for structures with diameters less than $10 \mathrm{~nm} \cdot{ }^{[22,23]}$ Thus, size effects are not likely to play a crucial role in determining the observed bandgaps of the InN nanobelts since the widths of our nanobelts are still larger than $10 \mathrm{~nm}$.

\section{Conclusions}

Single-crystalline InN nanobelts with a hexagonal wurtize structure have been successfully synthesized on SiN-coated $\mathrm{Si}$ substrates using a GSCVD technique. The as-prepared InN nanobelts are found to grow exclusively along the [110] direction, and the side surfaces are enclosed only by $\pm(001)$ and $\pm(1 \overline{1} 0)$ planes. This self-selectivity of facets in the nanobelts can be attributed to differences in their surface energies. The observation of catalytic nanoparticles at the growth fronts of the nanobelts suggests that the growth of the InN nanobelts is likely dominated by the VLS mechanism. The PL spectra obtained at $20 \mathrm{~K}$ exhibits a sharp peak at $0.76 \mathrm{eV}$, which can be ascribed to band-to-band emission, with a FWHM of only $14 \mathrm{meV}$, the narrowest value reported to date for InN materials. 


\section{Experimental}

The synthesis of InN nanobelts was carried out in a quartz-tube (25 mm outer diameter) furnace. A thin a-SiN layer $(200 \mathrm{~nm})$ was first deposited on $\mathrm{Si}(100)$ by low-pressure chemical vapor deposition (LPCVD). Thin gold catalyst films $(3 \mathrm{~nm})$ were then deposited on the a-SiN-coated Si substrates by direct-current (DC) sputtering (EMITECH, K550X). Pure indium powders (ACROS, 99.999\%) were placed in an alumina boat to provide the indium vapor source. The precoated substrate was placed face-up on top of the alumina boat, at a height approximately in line with the axis of quartz tube, and was subsequently transferred to the center of the quartz tube. We employed a new method for supplying $\mathrm{NH}_{3}$, namely, a guided-stream technique, wherein an additional small quartz tube $(10 \mathrm{~mm}$ in diameter) was inserted into the central zone of the main quartz tube reactor where the substrate was located. This guided-stream technique allowed for accelerated flow of $\mathrm{NH}_{3}$ during growth. Prior to growth, the quartz tube was degassed and purged with $\mathrm{NH}_{3}$ (50 standard cubic centimeters per minute $(\mathrm{sccm}))$. During the growth period, the furnace was maintained at $560^{\circ} \mathrm{C}$ for $3 \mathrm{~h}$. Previously, we had reported the synthesis of InN nanorods and nanowires using a similar setup without a guided stream. It was seen that the as-grown samples exhibited two different colors on different regions of the substrate, and this non-uniformity was attributed to variations in the temperature and concentration of the $\mathrm{NH}_{3}$ gas during transport [7].

The surface morphology of the nanobelts was observed using FESEM (JEOL 6700). The uniformity of the GSCVD-prepared products is greatly improved over that of the previously reported nanostructures produced by simple thermal CVD [7]. Raman spectroscopy (Renishaw 2000 microRaman spectrometer), XRD (Toshiba, A-40-Cu), and HRTEM (JEOL, JEM-4000EX) were employed to investigate the microstructure of the nanobelts. PL studies were performed at $20 \mathrm{~K}$ using the $488 \mathrm{~nm}$ line of an $\mathrm{Ar}^{+}$laser as the excitation source. An In$\mathrm{GaAs}$ detector was used to record the spectra in the infrared range.

Received: August 18, 2005

Final version: September 9, 2005 Published online: December 8, 2005

[1] S. N. Mohammad, H. Morkoc, Prog. Quantum Electron. 1996, 20, 361.

[2] J. Wu, W. Walukiewicz, K. M. Yu, J. W. Ager III, E. E. Haller, H. Lu, W. J. Schaff, Y. Saito, Y. Nanishi, Appl. Phys. Lett. 2002, 80, 3967.

[3] A. G. Bhuiyan, A. Hashimoto, A. Yamamoto, J. Appl. Phys. 2003, 94 , 2779.

[4] Y. J. Bai, Z. G. Liu, X. G. Xu, D. L. Cui, X. P. Hao, X. Feng, Q. L. Wang, J. Cryst. Growth 2002, 241, 189.

[5] a) O. Briot, B. Maleyre, S. Ruffenach, Appl. Phys. Lett. 2003, 83, 2919. b) O. Briot, B. Maleyre, S. Ruffenach, B. Gil, C. Pinquier, F. Demangeot, J. Frandon, J. Cryst. Growth 2004, 269, 22. c) B. Maleyre, O. Briot, S. Ruffenach, J. Cryst. Growth 2004, 269, 15.

[6] a) C. H. Liang, L. C. Chen, J. S. Hwang, K. H. Chen, Y. T. Hung, Y. F. Chen, Appl. Phys. Lett. 2002, 81, 22. b) J. Zhang, B. Xu, F. H. Jiang, Y. D. Yang, J. P. Li, Phys. Lett. A 2005, 337, 121. c) J. Zhang, L. Zhang, X. Peng, X. F. Wang, J. Mater. Chem. 2002, 12, 802. d) K. Sardar, F. L. Deepak, A. Govindaraj, M. M. Seikh, C. N. R. Rao, Small 2005, 1, 91. e) M. C. Johnson, C. J. Lee, E. D. BourretCourchesne, S. L. Konsek, S. Aloni, W. Q. Han, A. Zettl, Appl. Phys. Lett. 2004, 85, 5670. f) L. W. Yin, Y. Bando, Y. C. Zhu, D. Golberg, M. S. Li, Appl. Phys. Lett. 2004, 84, 1546. g) B. Schwenzer, L. Loeffler,
R. Seshadri, S. Keller, F. F. Lang, S. P. Denbaars, U. K. Mishra, J. Mater. Chem. 2004, 637.

[7] Z. H. Lan, W. M. Wang, C. L. Sun, S. C. Shi, C. W. Hsu, T. T. Chen, K. H. Chen, C. C. Chen, Y. F. Chen, L. C. Chen, J. Cryst. Growth 2004, 269, 87 .

[8] a) L. W. Yin, Y. Bando, D. Golberg, M. S. Li, Adv. Mater. 2004, 16 , 1833. b) S. D. Luo, W. Y. Zhou, W. X. Wang, Z. X. Zhang, L. F. Liu, X. Y. Dou, J. X. Wang, X. W. Zhao, D. F. Liu, Y. Gao, L. Song, Y. J. Xiang, J. J. Zhou, S. S. Xie, Appl. Phys. Lett. 2005, 87, 063109.

[9] a) S. D. Dingman, N. P. Rath, P. D. Markowitz, P. C. Gibbons, W. E. Buhro, Angew. Chem. Int. Ed. 2000, 39, 1470. b) H. Parala, A. Devi, F. Hipler, E. Maile, A. Birkner, H. W. Becker, R. A. Fischer, J. Cryst. Growth 2001, 231, 68. c) N. Takahashi, A. Niwa, T. Takahashi, T. Nakamura, M. Yoshika, Y. Momose, J. Mater. Chem. 2002, 12, 1573.

[10] Z. W. Pan, Z. R. Dai, Z. L. Wang, Science 2001, 291, 1947.

[11] a) J. C. Johnson, H. J. Choi, K. R. Knutsen, R. D. Schaller, P. Yang, R. J. Saykally, Nat. Mater. 2002, 1, 106. b) M. Huang, S. Mao, H. Feick, H. Yan, Y. Wu, H. Kind, E. Weber, R. Russo, P. Yang, Science 2001 292, 1897.

[12] Fundamentals of Optical Waveguides (Ed: K. Okamoto), Academic, San Diego, CA 2000

[13] H. Yan, J. Johnson, M. Law, R. He, K. Knutsen, J. R. McKinney, J. Pham, R. Saykally, P. Yang, Adv. Mater. 2003, 15, 1907.

[14] a) K. Xu, A. Yoshikawa, Appl. Phys. Lett. 2003, 83, 251. b) O. K Semchinova, J. Aderhold, J. Graul, A. Filimonov, H. Neff, Appl. Phys. Lett. 2003, 83, 5440. c) Y. Saito, H. Harima, E. Kurimoto, T. Yamaguchi, N. Teraguchi, A. Suzuki, T. Araki, Y. Nanishi, Phys. Status Solidi B 2002, 234, 796

[15] a) P. B. Hirsch, A. Howie, R. B. Nicholson, D. W. Pashley, M. L. Whelan, in Electron Microscopy of Thin Crystals (Ed: R. E. Krieger), Huntington, New York 1977, Ch.11. b) Y. Jiang, X. M. Meng, J. Liu, Z. Y. Xie, C. S. Lee, S. T. Lee, Adv. Mater. 2003, 15, 323.

[16] P. Yang, C. M. Leiber, J. Mater. Res. 1997, 12, 2981.

[17] a) R. S. Wagner, W. C. Ellis, Appl. Phys. Lett. 1964, 4, 89. b) Y. Ding, P. X. Gao, Z. L. Wang, J. Am. Chem. Soc. 2004, 126, 2066. c) C. Liang, Y. Shimizu, T. Sasaki, H. Umehara, N. Koshizaki, J. Phys. Chem. B 2004, 108, 9728. d) X. S. Fang, C. H. Ye, X. S. Peng, Y. H. Wang, Y. C. Wu, L. D. Zhang, J. Mater. Chem. 2003, 13, 3040.

[18] Z. L. Wang, Z. W. Pan, Z. R. Dai, Microsc. Microanal. Microstruct. 2002, 8,467 .

[19] CERIUS2 (Version 4.0, Accelrysis Inc.).

[20] J. Neugebauer, Phys. Status Solidi 2001, 227, 93.

[21] S. Gwo, C. L. Wu, C. H. Shen, W. H. Chang, T. M. Hsu, J. S. Wang, J. T. Hsu, Appl. Phys. Lett. 2004, 84, 3765.

[22] a) H. Cao, Y. G. Zhao, S. T. Ho, E. W. Seelig, Q. H. Wang, P. R. H. Chang, Phys. Rev. Lett. 1999, 82, 2278. b) J. H. Schön, Ch. Kloc, A. Dodabalapur, B. Batlogg, Science 2000, 289, 599. c) A. Serpengüzel, J. Opt. Soc. Am. B 2001, 18, 989.

[23] a) W. Zhang, N. Cue, K. M. Yoo, Opt. Lett. 1995, 20, 961. b) R. M. Balachandran, D. P. Pacheco, N. M. Lawandy, Appl. Opt. 1996, 35 , 640. c) W. L. Sha, C.-H. Liu, R. R. Alfano, Opt. Lett. 1994, 19, 1922. d) R. M. Balachandran, N. M. Lawandy, Opt. Lett. 1997, 22, 319. e) R. M. Balachandran, N. M. Lawandy, Opt. Lett. 1995, 20, 1271. f) M. A. Noginov, H. J. Caulfield, N. E. Noginova, P. Venkateswarlu, Opt. Commun. 1995, 118, 430.

[24] J. S. Huang, C. T. Wu, K. H. Chen, C. M. Chuang, L. C. Chen, T. T. Chen, Y. F. Chen, M. C. Lin, unpublished.

[25] V. Y. Davydov, A. A. Klochikhin, R. P. Seisyan, V. V. Emtsev, S. V. Ivanov, F. Bechstedt, J. Furthmüller, H. Harima, A. V. Mudryi, J. Aderhold, O. Semchinova, J. Graul, Phys. Status Solidi B 2002, 229 , R1. 